Supporting Information for

\title{
Probing Charge Injection-Induced Structural Transition in Ionic Liquids Confined at the $\mathrm{MoS}_{2}$ Surface
}

Wei Chen ${ }^{\dagger \dagger}$, Yumiao Lu ${ }^{\ddagger}, *$, Yanlei Wang ${ }^{\ddagger}$, Feng Huo ${ }^{\ddagger}$, Wei-Lu Ding, Li Wei ${ }^{\dagger}, *$ and Hongyan He ${ }^{\ddagger}$,*

$\dagger$ College of Light Industry and Chemical Engineering, Dalian Polytechnic University, Dalian 116034,

China

${ }^{\ddagger}$ CAS Key Laboratory of Green Process and Engineering, Beijing Key Laboratory of Ionic Liquids Clean

Process, Institute of Process Engineering, Chinese Academy of Sciences, Beijing 100190, China

Corresponding authors:

Yumiao Lu, Email: ymlv@ipe.ac.cn

Li Wei, Email: weili@dlpu.edu.cn

Hongyan He, Email: hyhe@ipe.ac.cn

Supporting Information includes 19 figures 

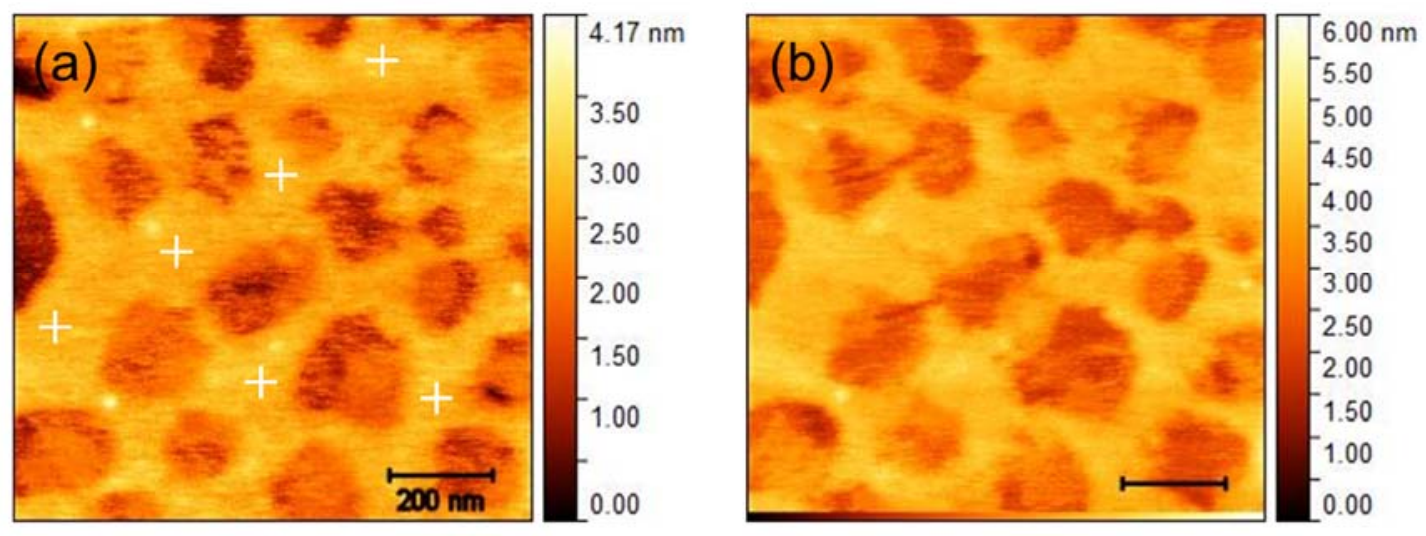

Fig. S1. AFM topography of $\left[\mathrm{C}_{12} \mathrm{mim}\right]\left[\mathrm{NTf}_{2}\right]$ at the $\mathrm{MoS}_{2}$ surface (a) before and (b) after indentation at a force of $50 \mathrm{nN}$ with the AFM tip of MESP-V2. The white crosses mark the indentation positions. 

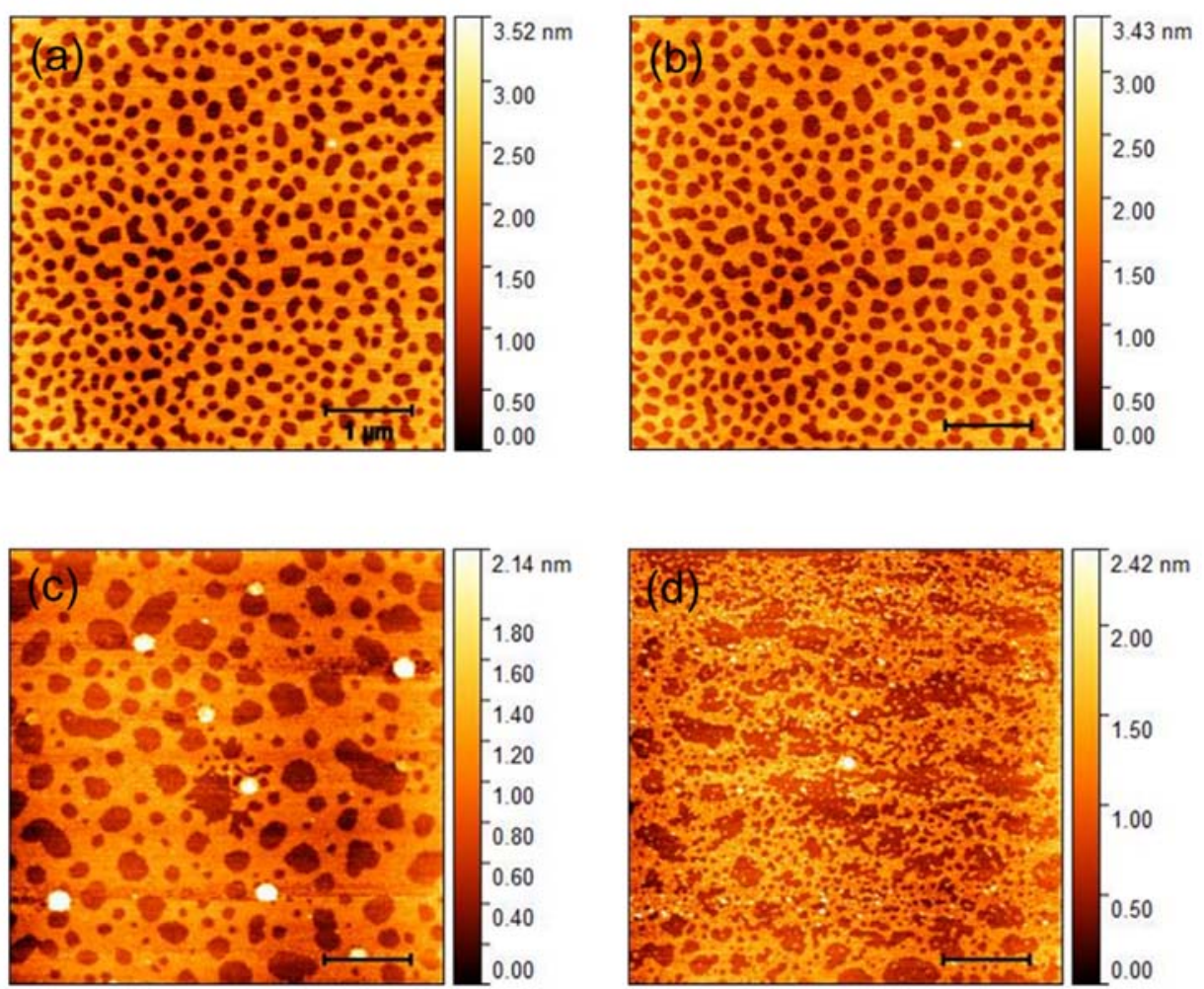

Fig. S2. AFM topography of $\left[\mathrm{C}_{12} \mathrm{mim}\right]\left[\mathrm{NTf}_{2}\right]$ at the $\mathrm{MoS}_{2}$ surface at (a) the first and (b) the fourth tapping mode scanning. AFM topography obtained in tapping mode (c) before and (d) after contact mode scanning at the normal force of $50 \mathrm{nN}$. The scale bars are $1 \mu \mathrm{m}$ in all panels. 

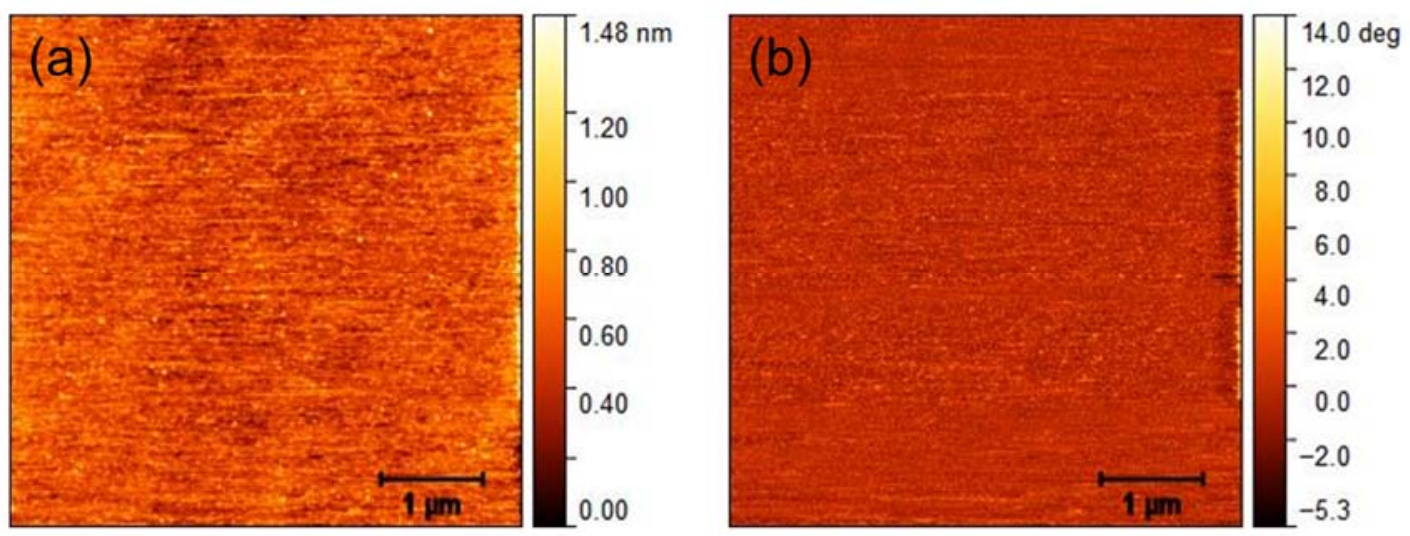

Fig. S3. (a) AFM topography and (b) the corresponding phase image of the pure solvent $\mathrm{CH}_{3} \mathrm{OH}$ at the $\mathrm{MoS}_{2}$ surface. 

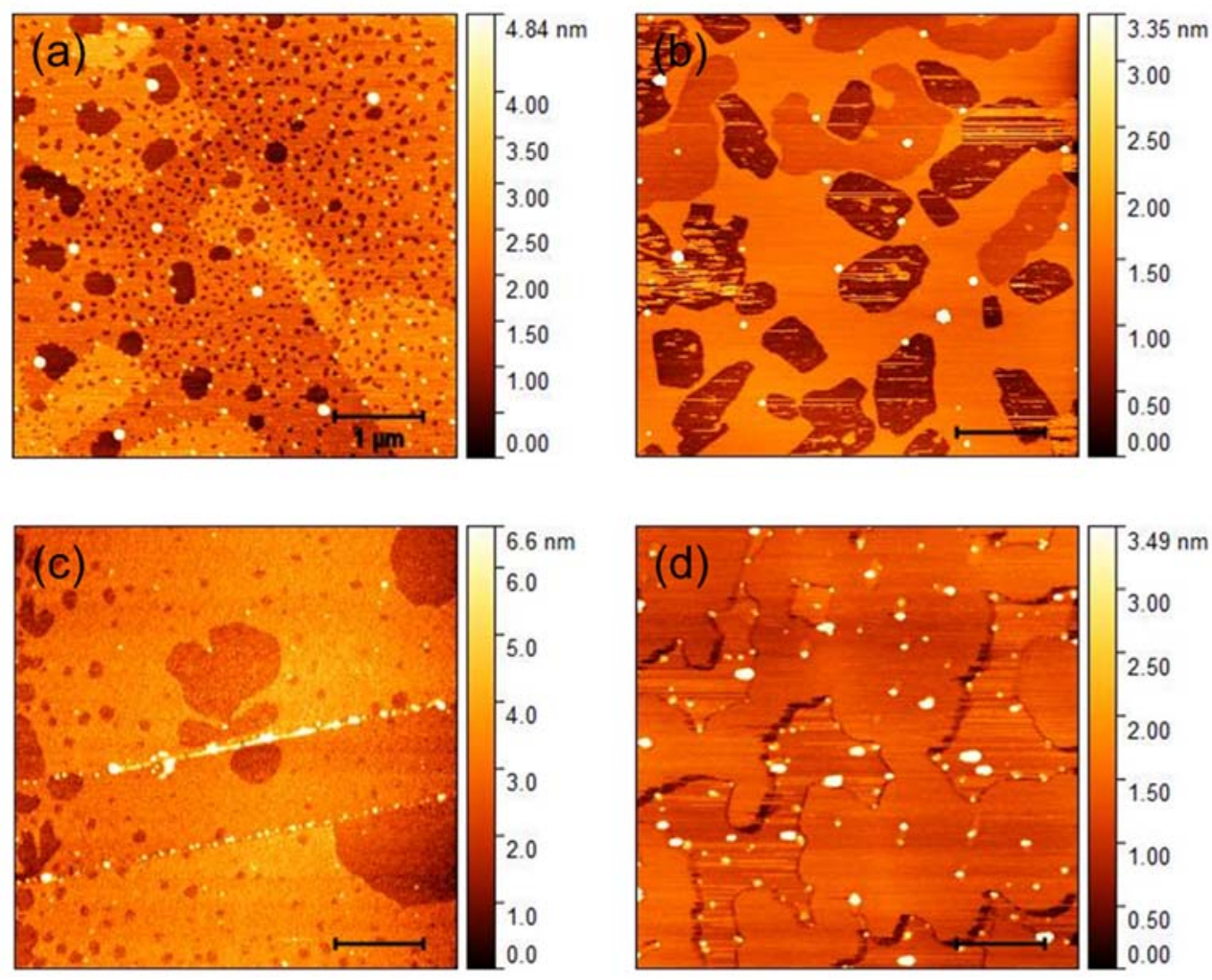

Fig. S4 AFM topography of $\left[\mathrm{C}_{12} \mathrm{mim}\right]\left[\mathrm{NTf}_{2}\right]$ at the $\mathrm{MoS}_{2}$ surface prepared under (a-b) ambient conditions and in (c-d) a glove box. 

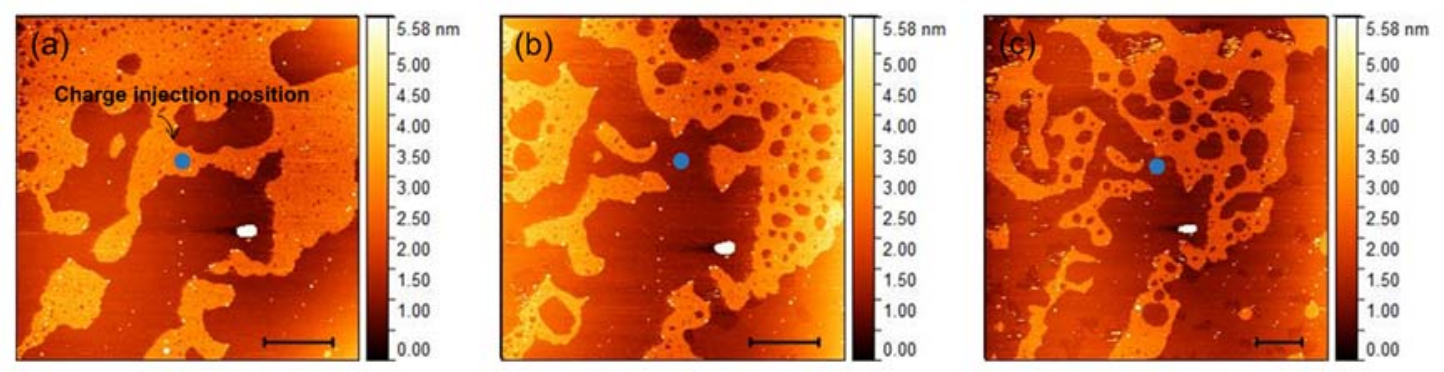

Fig. S5 Surface topography of the same region at (a) the initial state and (b-c) after positive charge injection with a tip voltage and time of $1 \mathrm{~V}-30 \mathrm{~s}$. The blue dots in (a-c) represent the charge injection position. The thickness of ILs in (a-c) is $\sim 1.4 \mathrm{~nm}, \sim 1.6$ $\mathrm{nm}$ and $\sim 1.5 \mathrm{~nm}$, respectively. The scale bars are $2 \mu \mathrm{m}$ in all panels. 

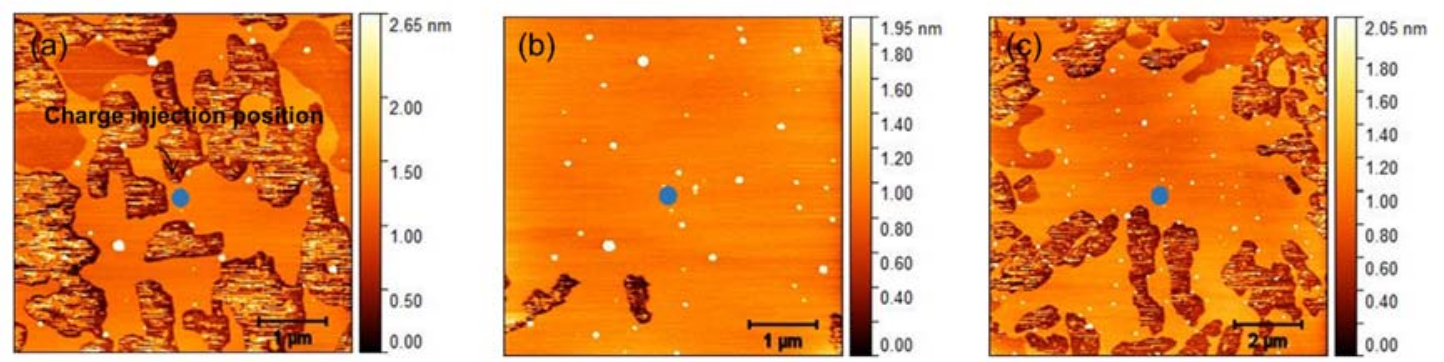

Fig. S6 Surface topography of the same region at (a) the initial state and (b-c) after positive charge injection with a tip voltage and time of $3 \mathrm{~V}-60 \mathrm{~s}$. The blue dots in (a-c) represent the charge injection position. 

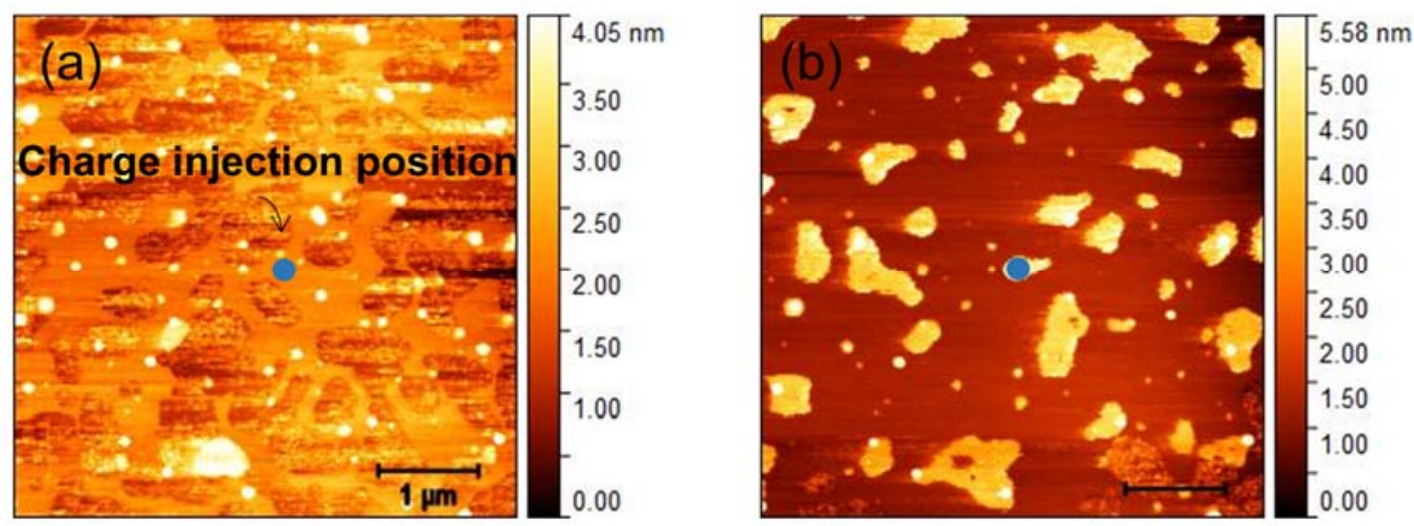

Fig. S7 Surface topography of the same region at (a) the initial state and (b) after positive charge injection with a tip voltage and time of $3 \mathrm{~V}-60 \mathrm{~s}$ for the IL-MoS 2 sample prepared in a glove box. The blue dots in (a-b) represent the charge injection positions. 

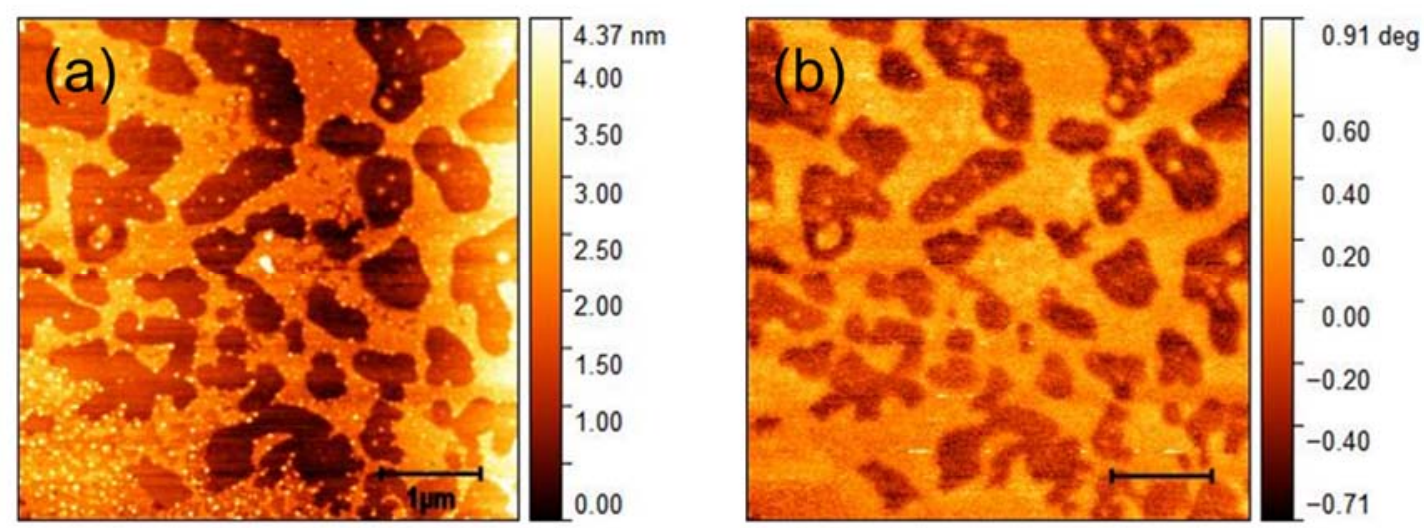

Fig. S8 (a) AFM topography and (b) the corresponding EFM phase image of IL-MoS2 after positive charge injection with a voltage and time of $1 \mathrm{~V}-60 \mathrm{~s}$. 

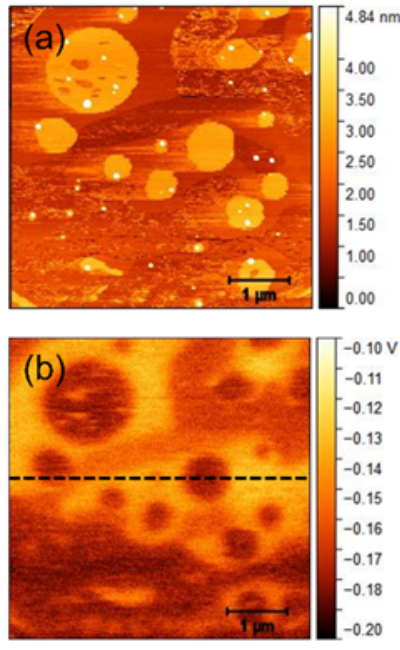

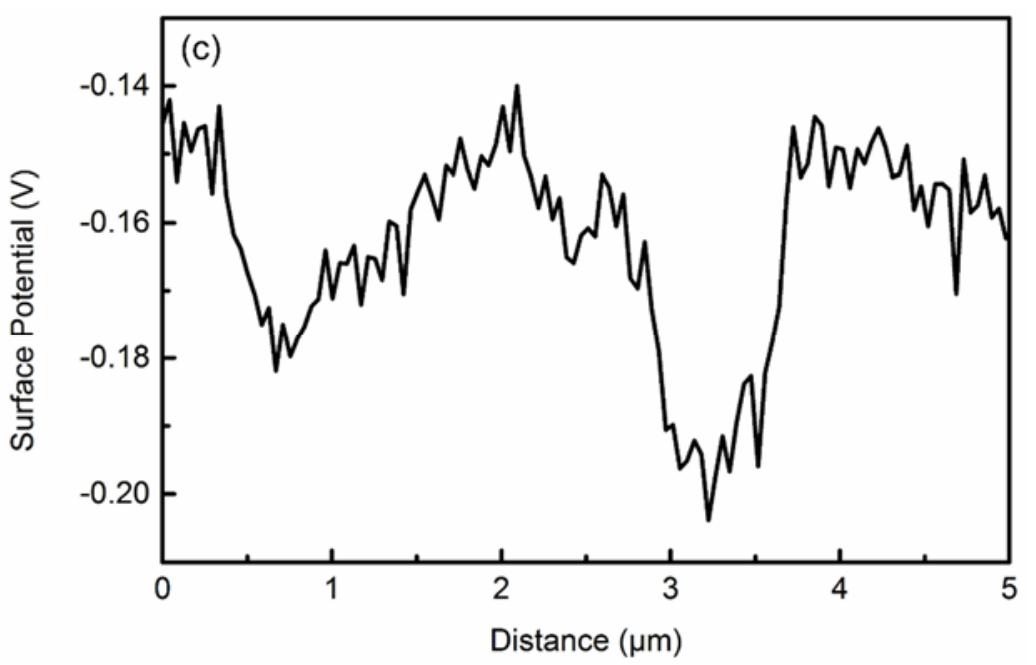

Fig. S9. (a) AFM topography and (b) the corresponding KPFM image of $\left[\mathrm{C}_{12} \mathrm{mim}\right]\left[\mathrm{NTf}_{2}\right]$ at the $\mathrm{MoS}_{2}$ surface along with (c) the potential change along the black dashed line in the KPFM image. 


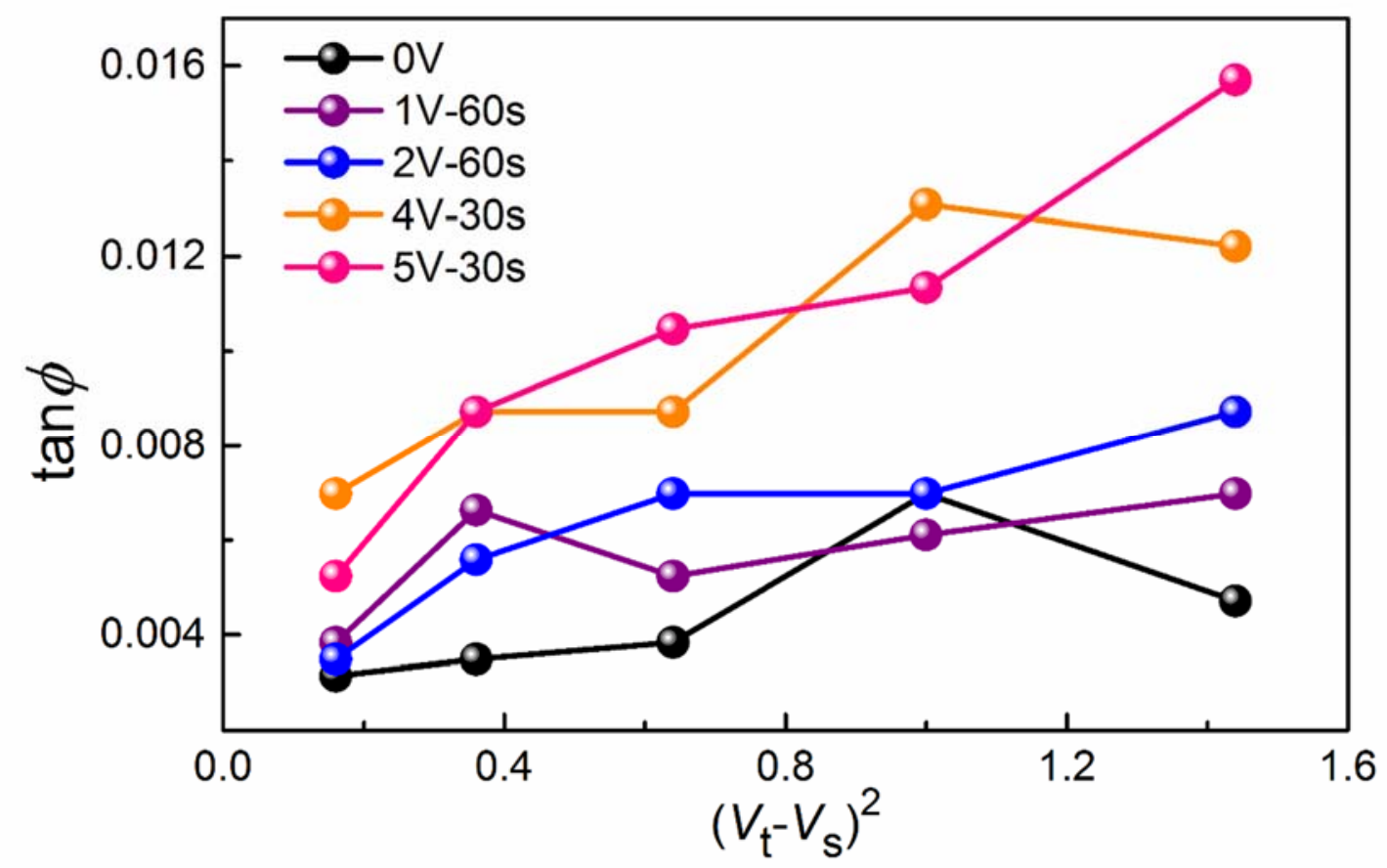

Fig. S10. Measured $\tan (\Phi)$ in the EFM image as a function of $\left(V_{\mathrm{t}}-V_{\mathrm{s}}\right)^{2}$ for $\mathrm{MoS}_{2}$ under positive charge injection. 


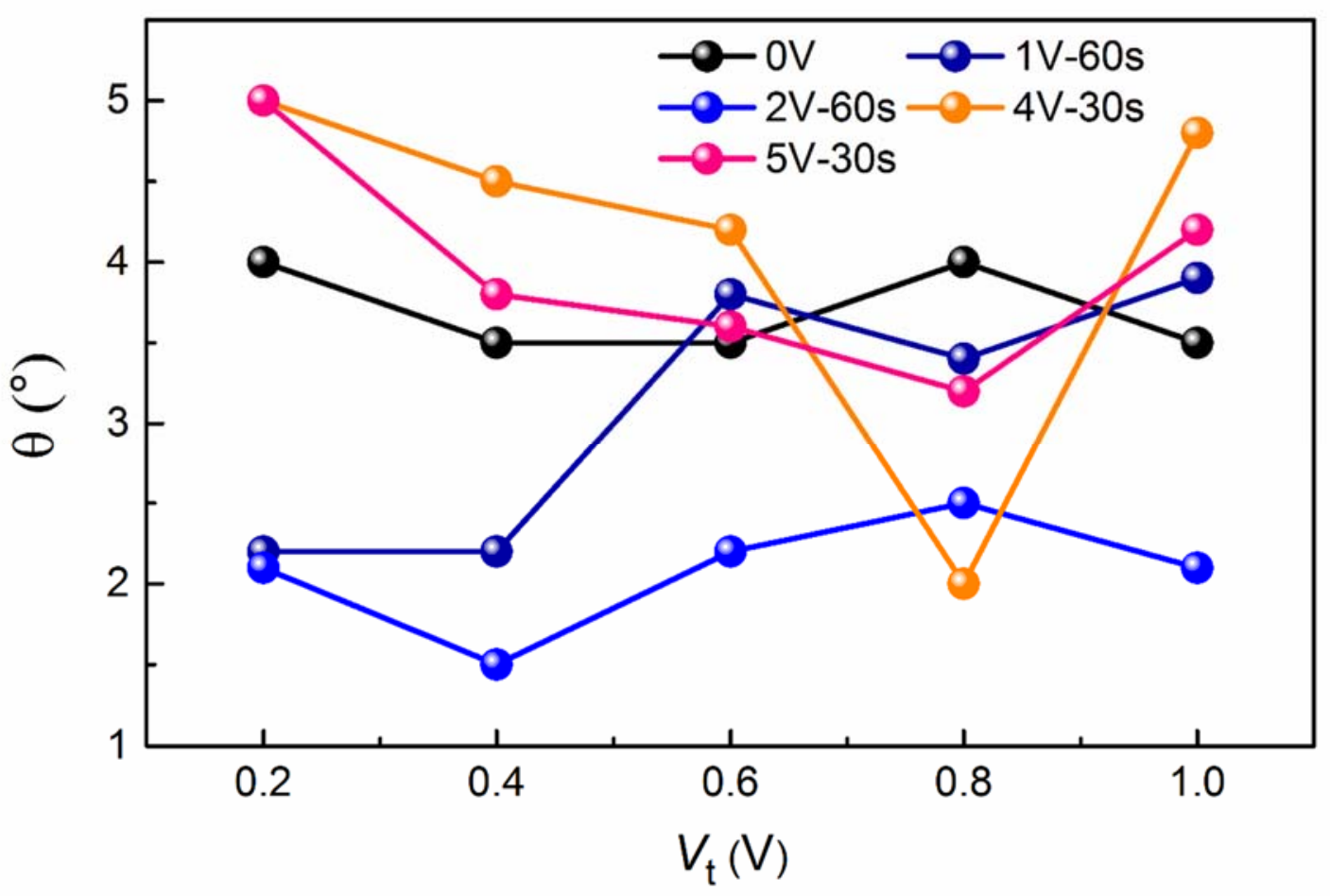

Fig. S11 Measured phase value $(\theta)$ of ILs as a function of tip bias. 

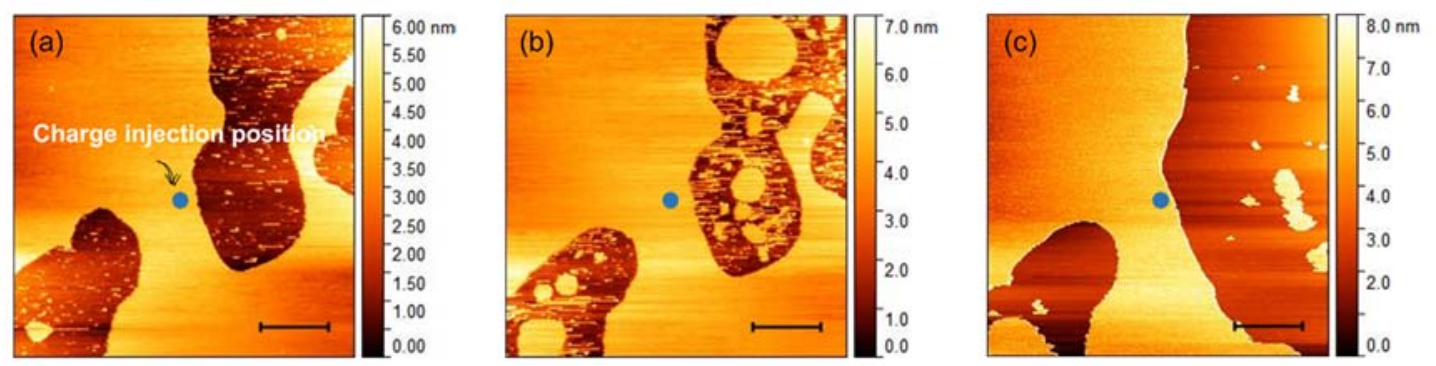

Fig. S12 Surface topography of the same region at (a) the initial state and after charge injection with tip voltages and contact times of (b) $-1 \mathrm{~V}-10 \mathrm{~s}$ and (c) $-1 \mathrm{~V}-30 \mathrm{~s}$. The blue dots in (a-c) represent the charge injection positions. The scale bars are $1 \mu \mathrm{m}$ in all panels. 


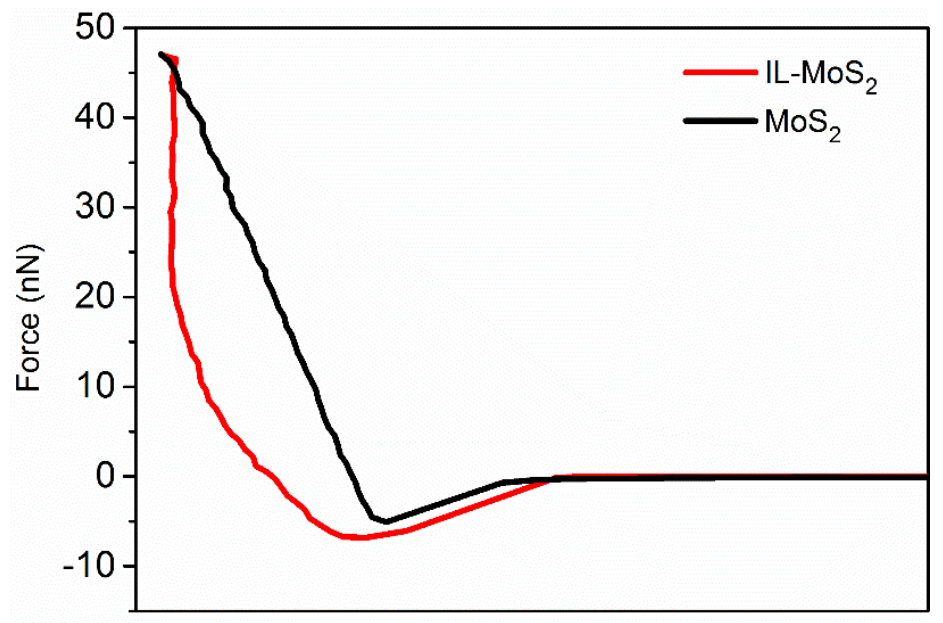

Tip-sample seperation

Fig. S13 Representative indentation curves obtained on IL-MoS 2 and $\mathrm{MoS}_{2}$ samples. 

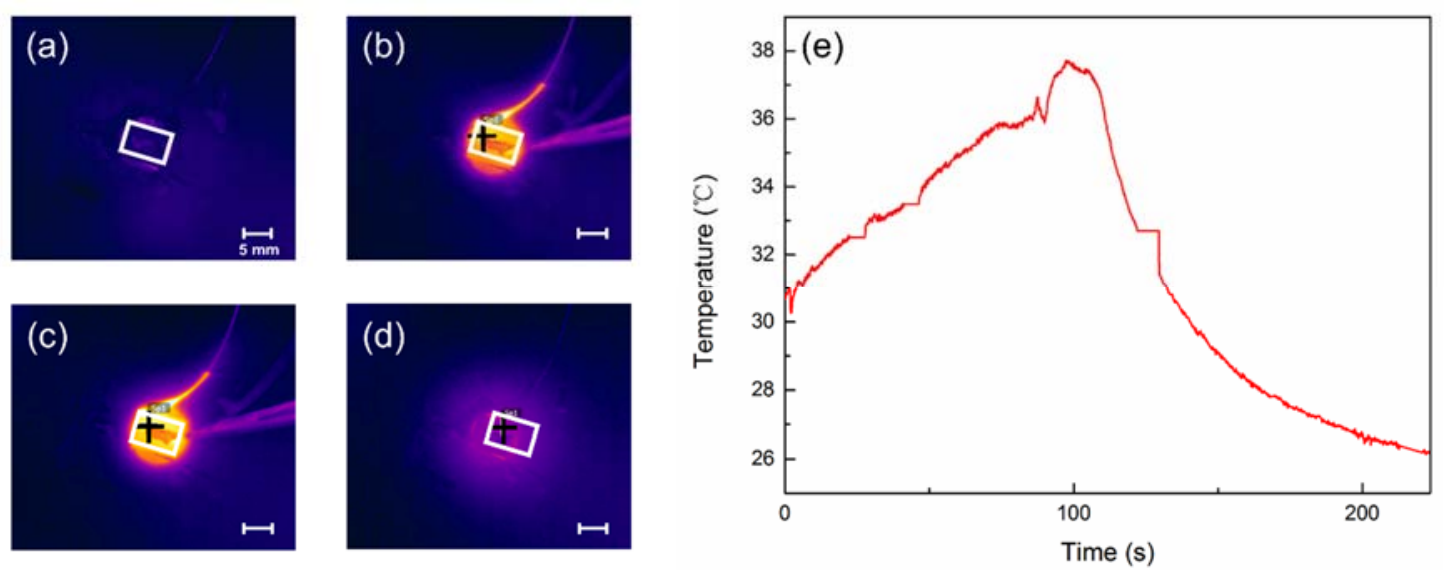

Fig. S14 (a-d) IR image of IL-MoS 2 when the applied voltage is $1.5 \mathrm{~V}$ and (e) the corresponding temperature change as a function of time. The rectangles in (a-d) mark the position of IL-MoS 2 sample. The scale bars are $5 \mathrm{~mm}$ in Fig.a-d. 

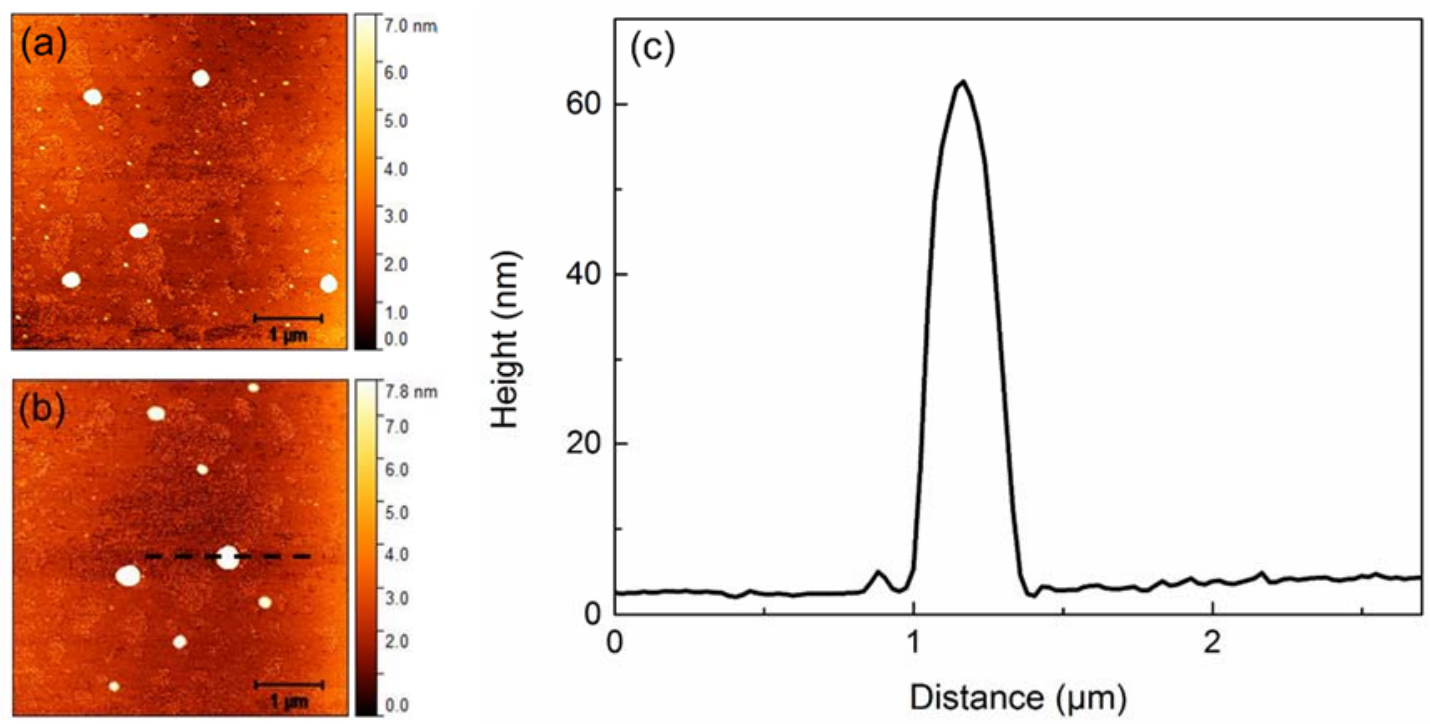

Fig. S15 (a-b) AFM topography images of the IL-MoS 2 sample after ex situ circuit experiments and (c) line profiles of the black dashed lines on the image. 

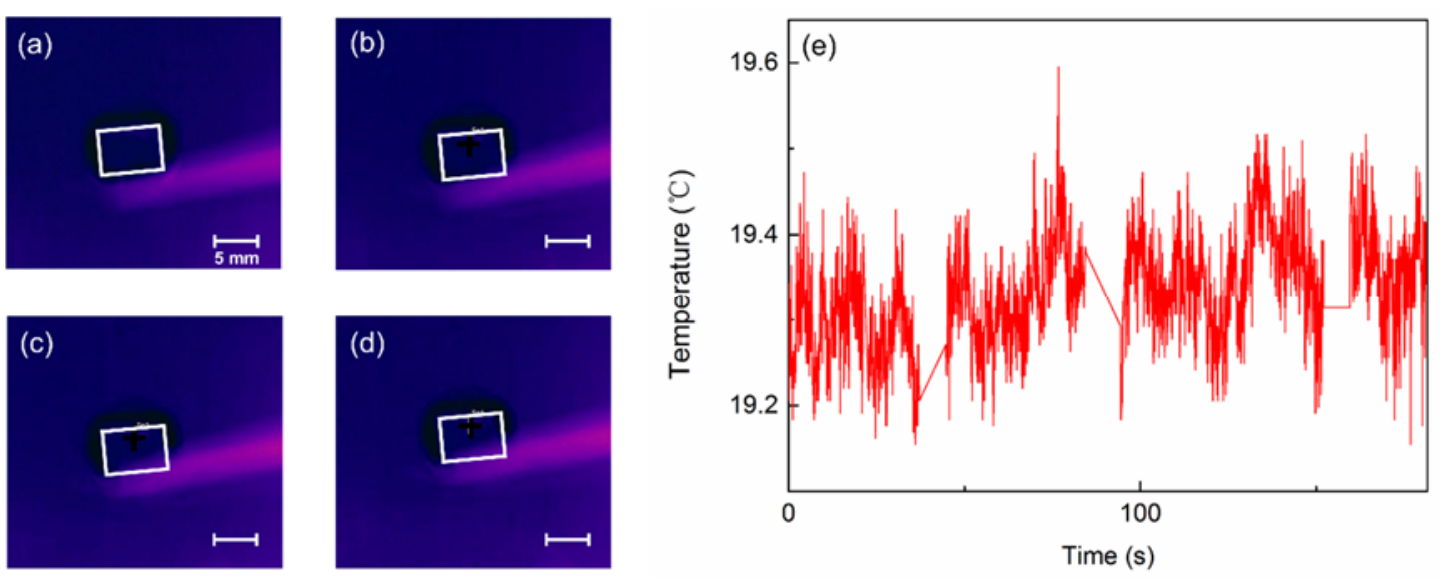

Fig. S16 (a-d) IR image of the IL-mica sample when the applied voltage is $1.5 \mathrm{~V}$ and (e) the corresponding temperature change as a function of time for the region marked by the white cross in the image. The rectangles in (a-d) mark the position of IL-mica sample. The scale bars are $5 \mathrm{~mm}$ in Fig.a-d. 

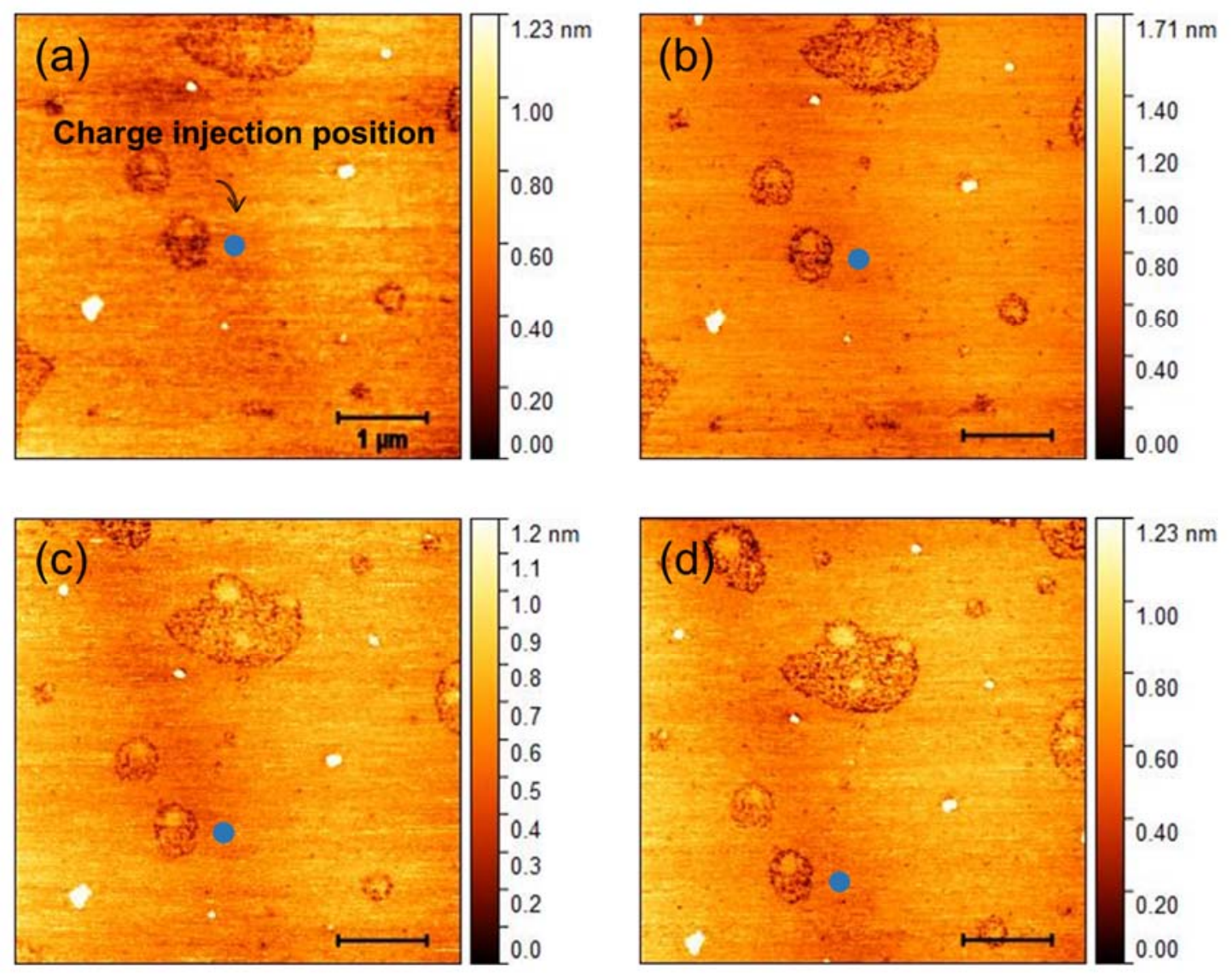

Fig. S17 Surface topography of the same region at (a) the initial state and after charge injection with tip voltages and contact times of (b) 1 V-30 s, (c) 2 V-30 s, and (d) 4 V$30 \mathrm{~s}$ for $\left[\mathrm{C}_{12} \mathrm{mim}\right]\left[\mathrm{NTf}_{2}\right]$ on the mica surface. The blue dots in (a-d) represent the charge injection positions. The scanning area is slightly deviated from the original position due to thermal drift. The scale bars are $1 \mu \mathrm{m}$ in all panels. 

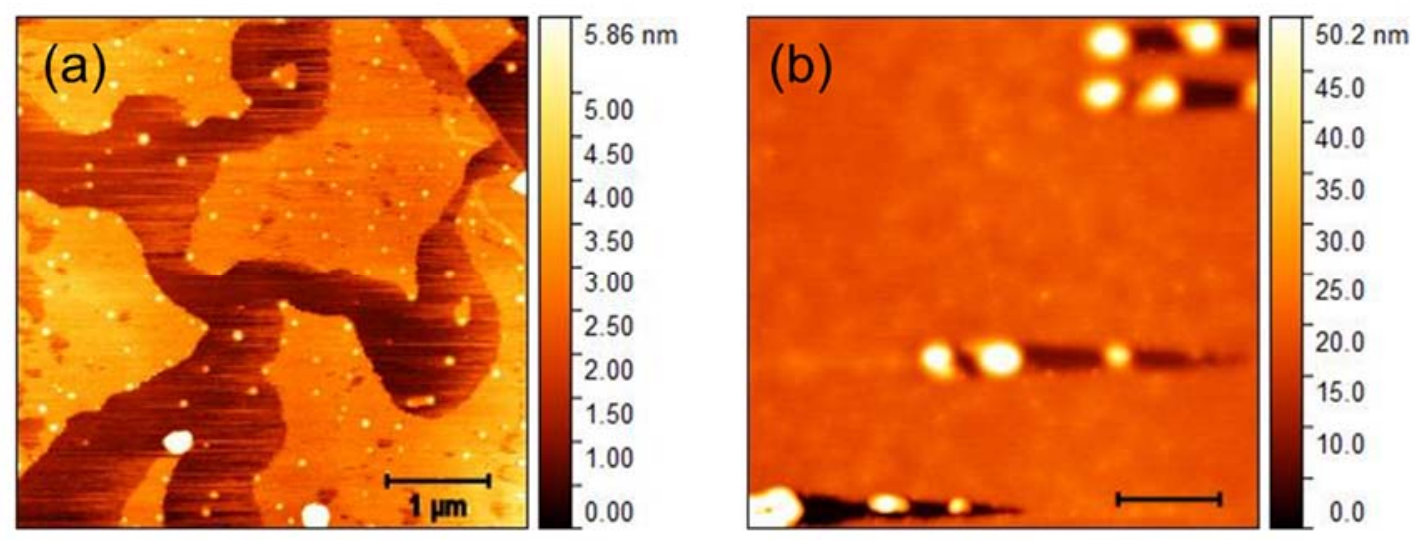

Fig. S18 AFM topography images of the IL-MoS 2 sample (a) before and (b) after annealing treatment at $50{ }^{\circ} \mathrm{C}$ for $9 \mathrm{~min}$. 

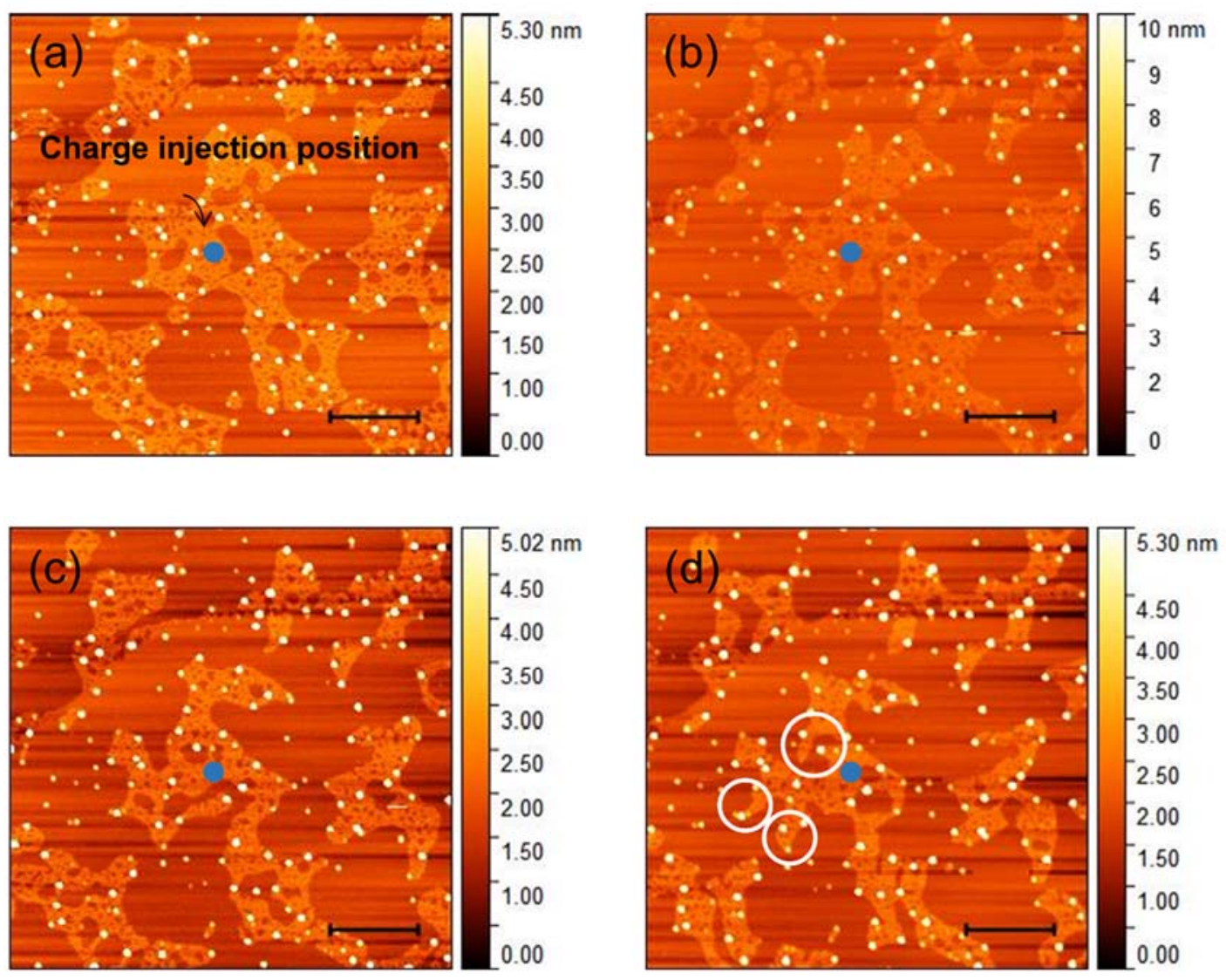

Fig. S19 And surface topography of the same region at (a) the initial state, (b) after EFM measurements with a tip bias of $2 \mathrm{~V}$, and after charge injection with tip voltages and contact times of (c) 2 V-30 s and (d) 5 V-30 s. The blue dots in (a-d) represent the charge injection positions. The white circles in (d) mark the positions where obvious structural change occurs. The scale bars are $1 \mu \mathrm{m}$ in all panels. 